\title{
Attractiveness of black and white modified Shannon traps to phlebotomine sandflies (Diptera, Psychodidae) in the Brazilian Amazon Basin, an area of intense transmission of American cutaneous leishmaniasis
}

\author{
Andreia Fernandes Brilhante ${ }^{1, *}$, Márcia Moreira de Ávila ${ }^{2}$, Jailson Ferreira de Souza ${ }^{3}$, \\ Antônio Ralph Medeiros-Sousa ${ }^{1}$, Priscila Bassan Sábio ${ }^{1}$, Marcia Bicudo de Paula ${ }^{1}$, \\ Rodrigo Espindola Godoy ${ }^{1}$, Leonardo Augusto Kohara Melchior ${ }^{1,4}$, Vânia Lúcia Brandão Nunes ${ }^{5}$, \\ Cristiane de Oliveira Cardoso ${ }^{4}$, and Eunice Aparecida Bianchi Galati ${ }^{1}$ \\ 1 Faculty of Public Health, University of São Paulo, São Paulo, Brazil \\ 2 Federal Institute of Acre, Rio Branco, Acre, Brazil \\ 3 Management of Endemics, City Hall of Xapuri, Acre, Brazil \\ ${ }^{4}$ Federal University of Acre, Rio Branco, Acre, Brazil \\ 5 Laboratory of Human Parasitology, Anhanguera-Uniderp University, Campo Grande, Mato Grosso do Sul, Brazil
}

Received 1 January 2017, Accepted 20 May 2017, Published online 8 June 2017

\begin{abstract}
In the Amazon region the phlebotomine fauna is considered one of the most diverse in the world. The use of Shannon traps may provide information on the anthropophily of the species and improve the traps' performance in terms of diversity and quantity of insects collected when white and black colored traps are used together. This study sought to verify the attractiveness of the traps to the phlebotomine species of the Brazilian Amazon basin using Shannon traps under these conditions. The insects were collected using two Shannon traps installed side by side, one white and the other black, in a primary forest area of the municipality of Xapuri, Acre, Brazil. Samples were collected once a month during the period August 2013 to July 2015. A sample of females was dissected to test for natural infection by flagellates. A total of 6,309 (864 males and 5,445 females) specimens ( 36 species) were collected. Psychodopygus carrerai carrerai (42\%), Nyssomyia shawi (36\%), and Psychodopygus davisi (13\%), together represented $90 \%$ of the insects collected. Nyssomyia shawi and Psychodopygus davisi were more attracted by the white color. Specimens of Nyssomyia shawi, Nyssomyia whitmani, and Psychodopygus hirsutus hirsutus were found naturally infected by flagellates in the mid and hindgut. This is the first study in Acre state using and comparing both black and white Shannon traps, demonstrating the richness, diversity, and anthropophilic behavior of the phlebotomine species and identifying proven and putative vectors of the etiological agents of leishmaniasis.
\end{abstract}

Key words: Phlebotomine, Attraction, Shannon traps, Anthropophily, American cutaneous leishmaniasis, Amazonia.

\begin{abstract}
Résumé - Attractivité pour les phlébotomes (Diptera: Psychodidae) des pièges Shannon modifiés de couleur noire et blanche dans le bassin amazonien brésilien, zone de transmission intense de la leishmaniose cutanée américaine. Dans la région amazonienne, la faune des phlébotomes est considérée comme l'une des plus diversifiées au monde. L'utilisation de pièges Shannon peut fournir des informations sur l'anthropophilie de l'espèce et améliorer les performances des pièges en termes de diversité et de quantité d'insectes recueillis lorsque des pièges de couleur blanche et noire sont utilisés ensemble. Cette étude a cherché à vérifier l'attractivité des pièges pour les phlébotomes du bassin de l'Amazone brésilien en utilisant des pièges Shannon dans ces conditions. Les insectes ont été recueillis en utilisant deux pièges Shannon installés côte à côte, l'un blanc et l'autre noir, dans une zone de forêt primaire de la municipalité de Xapuri, Acre, au Brésil. Des échantillons ont été prélevés une fois par mois pendant la période d'août 2013 à juillet 2015. Un échantillon de femelles a été disséqué pour la recherche d'une infection naturelle par des flagellés. Un total de 6309 spécimens (864 mâles et 5445 femelles) de 36 espèces ont été recueillis dans les pièges. Psychodopygus carrerai carrerai (42\%), Nyssomyia shawi (36\%) et Psychodopygus davisi (13\%) représentent ensemble $90 \%$ des insectes récoltés.
\end{abstract}

\footnotetext{
* Corresponding author: brilhanteaf@usp.br
} 
Nyssomyia shawi et Psychodopygus davisi étaient plus attirés par la couleur blanche. Des spécimens de Nyssomyia shawi, Nyssomyia whitmani et Psychodopygus hirsutus hirsutus ont été trouvés naturellement infectés par des flagellés dans l'intestin moyen et postérieur. Ceci est la première étude réalisée dans l'état d'Acre à l'aide de pièges Shannon noirs et blancs, qui démontre la richesse, la diversité, le comportement anthropophile des espèces de phlébotomes et qui identifie des vecteurs confirmés et putatifs des agents étiologiques de la leishmaniose.

\section{Introduction}

Phlebotomine flies (Diptera: Psychodidae) are holometabolous insects that are of medical interest mainly because of their involvement in the transmission of etiological agents of leishmaniasis. Currently, in the Neotropics, 520 species have been described and about half of these occur in Brazil [27]. In the Amazon region, the phlebotomine fauna is rich and considered one of the most diverse in the world, with the genera Psychodopygus, Nyssomyia, and Trichophoromyia as the most abundant in entomological surveys [30, 52, 66]. Furthermore, this fauna has a wide variety of incriminated and proven vectors acting in the transmission of leishmaniasis agents, which are commonly represented by different Leishmania species, particularly those of the subgenus Viannia [42].

The use of different types of traps may provide some information about the diversity of insect species in all kinds of environments. In 1939, Shannon [61] developed a trap using white fabric with light and animal bait to attract mosquitoes. Over the years, and with some modifications, the trap has been used in studies of mosquitoes and phlebotomines with the objective of verifying their anthropophily [46].

The attraction of insects to color was described by Browne and Bennet [8] who found that mosquitoes are more attracted to dark than light colors. However, the species may respond differently to this attraction, as reported by Gilbert and Gouck [32] with regard to mosquitoes and Galati et al. [24, 25], Cruz et al. [17], Moschin et al. [48], and Infran et al. [38] to phlebotomine sandflies.

In Acre (AC) state, little is known about the phlebotomine fly fauna and its behavior. Studies on these insects, undertaken between 2008 and 2016, have only used CDC (Centers for Disease Control) light traps, and have shown a great diversity of species [3, 5, 64, 69]. However, no collections had ever been undertaken in the state with other types of traps, such as the Shannon.

On the basis of this information, and the significant prevalence of cutaneous leishmaniasis in Acre state and in Xapuri municipality (AC), this study sought to verify the phlebotomine species and the traps' attractiveness to them in the Amazonian forest of the Acre basin, using white and black Shannon traps.

\section{Methods}

\section{Study area}

The study was conducted in a rural area about $175 \mathrm{~km}$ from Rio Branco, the Acre state capital, in Xapuri municipality, where human and canine cases of American cutaneous leishmaniasis (ACL) have been reported. The municipality is situated in the Vale do Acre mesoregion, and is bordered to the north by the state capital, to the south by Epitaciolândia municipality; to the east by Capixaba and Bolivia; to the west by Brasiléia (Fig. 1) [1,37]. The primitive vegetation of Xapuri consists of the Amazon biome characterized by a tropical climate with abundant rainfall from October to April and dry months between May and September [19]. The average annual temperature is $27^{\circ} \mathrm{C}$. The human population comprises around 16,000 inhabitants [1, 37].

Xapuri attracts tourists from different parts of the world, motivated by the historical and environmental context which has made the municipality the scene of great conflicts led by the Chico Mendes activist. In addition, it has an ecolodge located in Seringal Cachoeira about $32 \mathrm{~km}$ from the urban area of Xapuri, where ecological and environmental tourism activities such as hiking and tree climbing (known as "Arvorismo") are proposed. This is considered to be the largest circuit in the Amazon, with $600 \mathrm{~m}$ in length. The local vegetation consists of native plants and large trees such as Bertholletia excelsa, Hevea brasiliensis, and Ceiba pentandra. Ceiba pentandra is popularly known as "Samaúma" and one specimen has become a tourist attraction. More than 500 years old, it is the largest tree in the region at approximately $35 \mathrm{~m}$ in height, with a diameter of $25 \mathrm{~m} \mathrm{[73].}$

\section{Phlebotomine collections}

White and black Shannon traps similar to those described by Galati et al. [25] were installed in a primary forest area, side by side at the same distance, about $3 \mathrm{~m}$, from "Samaúma". The choice of this collection area was due to previous observations of fieldwork, indicating that the Samaúma is a shelter for sandflies (Fig. 2). The sandflies were collected once a month between August 2013 and July 2015, from $6 \mathrm{pm}$ to $10 \mathrm{pm}$. However, in the months of March 2014, August 2014, April 2015, and July 2015 the collections extended for $24 \mathrm{~h}$ uninterrupted. In October 2013, May 2014, and February 2015, due to heavy rains that made it impossible to access the area, the collections were not performed.

Each trap was illuminated at night with a fixed LED (lightemitting diode) light and manual flashlights. The captures were performed using Castro aspirators by two individuals circulating at the same time between the two traps. When the 24-h collections were carried out, the individuals were replaced by other collectors every $6 \mathrm{~h}$. The insects were captured at twohour intervals and kept in separate vials. They were then taken to the field laboratory, where the vials were opened in a nylon cage $(30 \mathrm{~cm} \times 30 \mathrm{~cm} \times 30 \mathrm{~cm})$. Then, female specimens were recaptured and dissected to investigate the presence of 


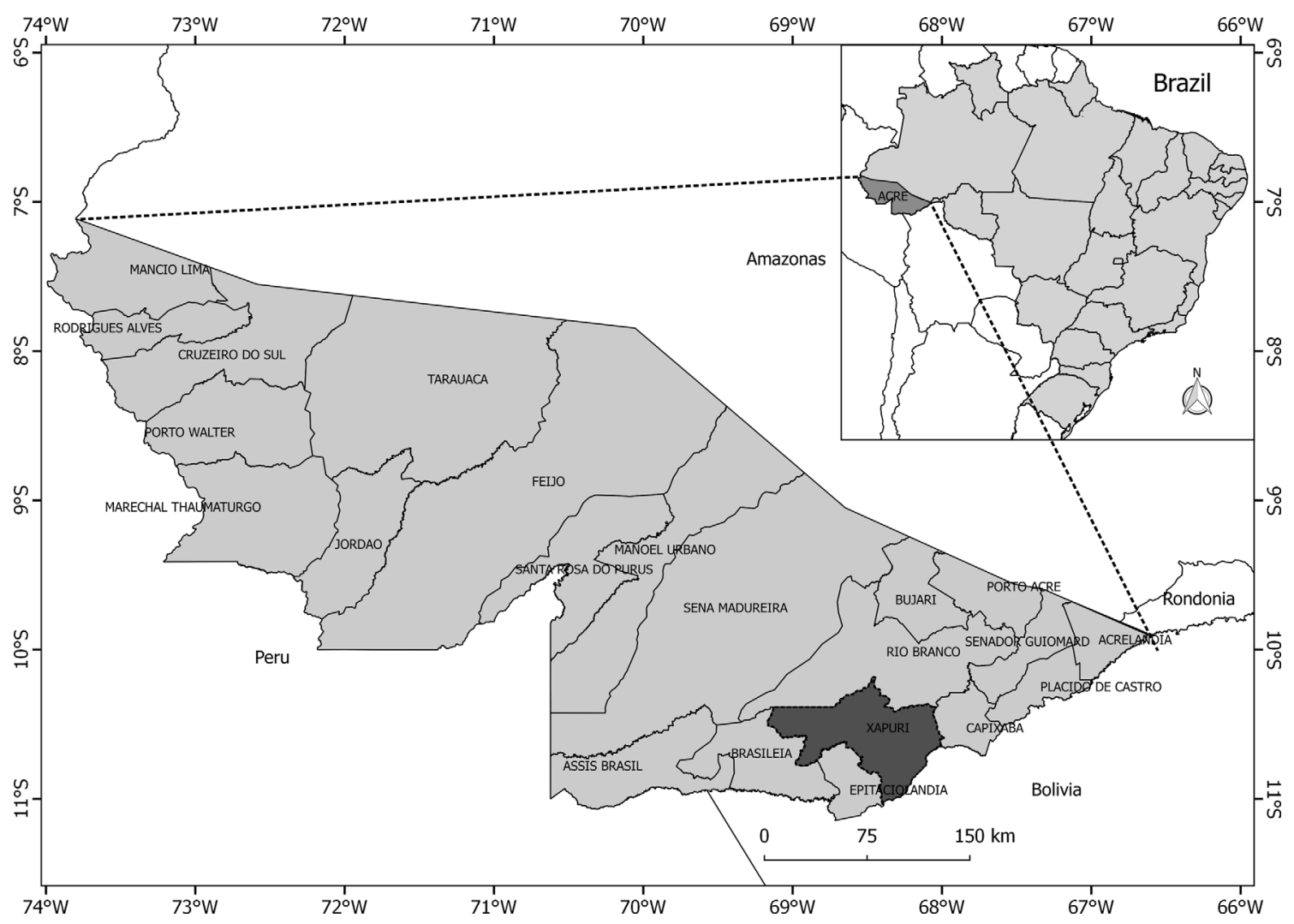

Figure 1. Political map of Brazil, highlighting the Acre state and Xapuri municipality with the study area.

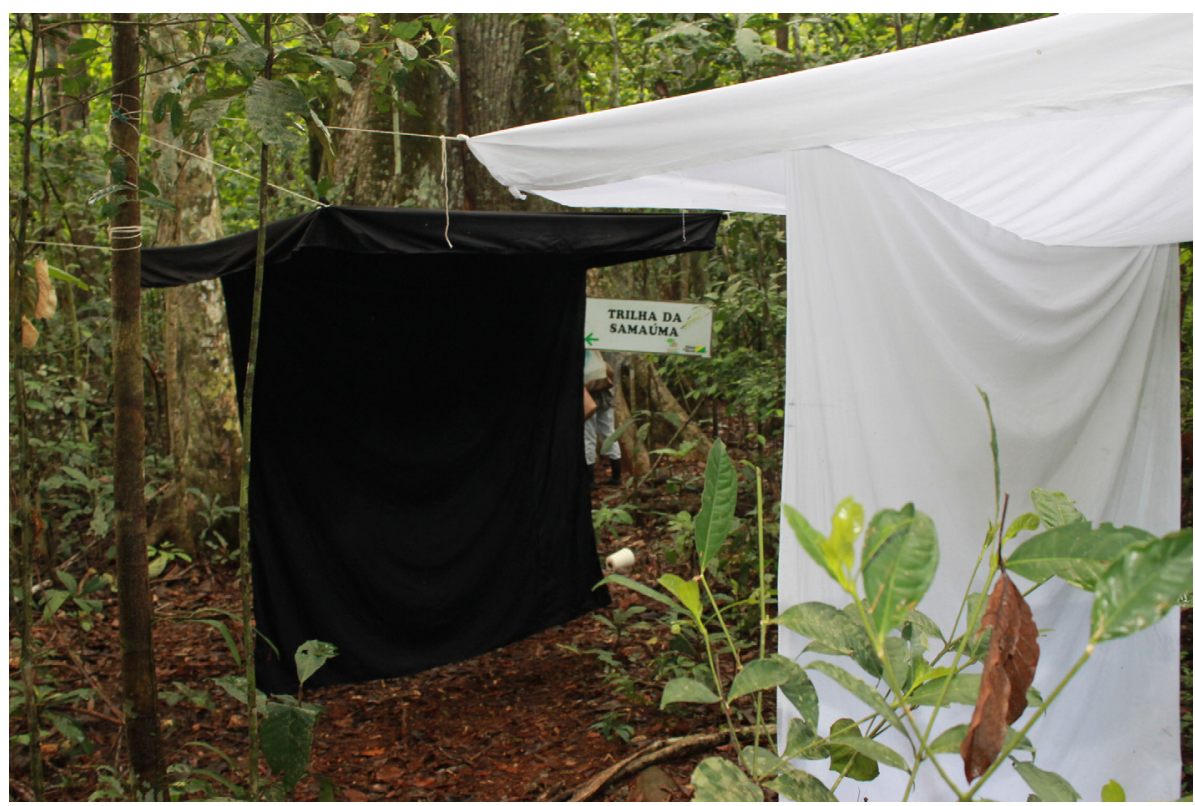

Figure 2. The black and white modified Shannon traps situated in front of the "Samaúma" and near the track area, Xapuri municipality, Acre State, Brazil.

flagellates in their guts. The females were immobilized with ethyl acetate and dissected on slides in a drop of sterile saline solution. After their legs and wings were removed, the insects were transferred to another drop of sterile saline. Afterwards, the gut and spermathecae were exposed, covered by a coverslip, and examined under the microscope $(400 \times)$ to test for natural infection by flagellates and to identify the phlebotomine species. The undissected females and the males were clarified 
for identification in accordance with Galati's keys [26] and complementary bibliography $[33,50,57,58]$. A sample of these clarified insects was mounted in Nelson Cerqueira (NC) resin between slide and coverslip. The remaining insects were examined in eugenol. The abbreviations of the species' names follow Marcondes [45].

\section{Data analysis}

To confirm sample sufficiency and species richness, the EstimateS program version 9.1.0 was used to plot 1,000 (sample-based and individual-based) randomizations without any replacement and a 95\% confidence interval [15].

The hourly rhythm of 24-h collections in dry and rainy months was demonstrated by the proportion rate. The monthly distribution of the species was calculated by Williams' geometric mean [35].

Shapiro-Wilk normality tests were applied for the abundance data; as none of the variables presented evidence of Gaussian distribution, non-parametric tests were used. A Spearman Rank-order Coefficient $\left(r_{\mathrm{s}}\right)$ test was used to analyze the correlation between the three most abundant species and the rainfall. The precipitation data used in this analysis were those of Rio Branco (AC) for a chronological series of the period 1970-2014, obtained by the Civil Defense of the State of Acre. This is because there are no complete data on climatic variables in Xapuri, nor from the nearest meteorological station, Epitaciolândia (AC).

To compare the attractiveness of the traps to the sandflies and the periods of collection (2013-2014 and 2014-2015), a Mann-Whitney test was used for $N \geq 10$. The significance level in all statistical tests was considered to be $p<0.05$. The rate of natural infection by flagellates was calculated according to the formula given by Paiva et al. [51].

\section{Results}

A total of 6,309 specimens (864 males and 5,445 females) were attracted to the two traps, constituting a species richness of 36, distributed among four subtribes and 10 genera. The frequencies of the species and female/male ratios collected in the white and black traps are shown in Table 1. Psychodopygus carrerai carrerai (41.90\%), Nyssomyia shawi (36.00\%), and Psychodopygus davisi (12.52\%) predominated, the three together accounting for $90.42 \%$ of the insects collected.

The species attracted to the black trap were different from those attracted to the white one; three species: Ps. paraensis, Ps. sp. (Guyanensis series), and Th. ubiquitalis were collected exclusively on the white trap and six: Ev. bacula, Ev. tarapacaensis, Mi. trinidadensis, Ny. fraihai, $P a$. bigeniculata, and Pa. pifanoi exclusively on the black trap. The black trap attracted one more species than the white (Table 1).

Except for three species of the Trichophoromyia genus, Th. auraensis, Th. octavioi, and Th. ruifreitasi, whose females are indistinguishable, among the others attracted, Evandromyia bacula, Psathyromyia bigeniculata, and $P a$. pifanoi, the species were represented exclusively by males, while
11 species: Evandromyia tarapacaensis, Ev. saulensis, Ev. termitophila, Lutzomyia evangelistai, Lu. sherlocki, Micropygomyia trinidadensis, Nyssomyia fraihai, Psathyromyia aragaoi, Psychodopygus paraensis, Psychodopygus sp. (Chagasi series), and Psychodopygus sp. (Guyanensis series) were represented only by females. For the nine most frequent species $\geq 0.30 \%$, generally the females were more attracted than males to black and white traps. However, for both colors, the difference in attractiveness between sexes was statistically significant $(p<0.05)$ by the Mann-Whitney test only for Ny. shawi, Ps. hirsutus hirsutus, and Ps. llanosmartinsi, and to the white trap for $N y$. whitmani, Ps. davisi, and Ps. lainsoni (Table 2).

In relation to the color of the traps, the white one was more attractive to all species, but statistically significant by MannWhitney test $(p<0.05)$ only for Ny. whitmani and PS. davisi (Table 2).

The sample-based species accumulation curves in both traps were close to the asymptote. Both traps showed total overlap in their estimated confidence intervals for number of species, suggesting no differences in observed richness based on samples (Fig. 3). Although the white trap attracted 2.15 times more specimens than the black one (Table 1), the accumulation curve based on individuals shows the black trap having a tendency to attract more species than the white (Fig. 4).

In relation to the hourly rhythm of the most frequent species, Ny. shawi, Ps. carrerai carrerai, and Ps. davisi, the highest peak occurred in the rainy season between 18 and $20 \mathrm{~h}$, gradually diminishing, however, until 4-6 h. On the other hand, in the dry season, the two Psychodopygus species presented their highest peak between 20 and $22 \mathrm{~h}$. Practically no insect was collected during the day time, though in the interval between 18 and $22 \mathrm{~h}$, the three species became active again (Fig. 5).

The monthly distribution by Williams' geometric mean of the species Ny. shawi, Ps. carrerai carrerai, and Ps. davisi is shown in Figure 6. Nyssomyia shawi was more frequently collected in the months considered dry, while Ps. carrerai carrerai and Ps. davisi in the rainy months. No significant correlation was observed between the average number of specimens and rainfall for the species $N y$. shawi $\left(r_{\mathrm{s}}=-0.15\right.$; $p=0.49)$ and Ps. davisi $\left(r_{\mathrm{s}}=0.27 ; p=0.22\right)$. However, Ps. carrerai carrerai $\left(r_{\mathrm{s}}=0.44 ; p<0.05\right)$ presented a significant positive correlation.

Comparing the collection periods August 2013-July 2014 and August 2014-July 2015, it was found that in the former ( $n=4,239$ specimens) twice as many specimens were collected as in the second $(n=1,610)$. However, in the nine months in which there were collections in both periods, no significant difference was observed between the medians of insects attracted in each period $(U=25 ; p=0.19)$.

A total of 708 females were dissected. Nyssomyia shawi, Ny. whitmani, and Ps. hirsutus hirsutus were found naturally infected by flagellates in the mid and hind portion of the gut. Their flagellate infection rates were $0.44 \%, 10.00 \%$, and $7.14 \%$, respectively, with a rate of $0.56 \%$ in the total dissected females. Leishmania DNA was detected by polymerase chain reaction (PCR) in one specimen of Ps. hirsutus and another 
Table 1. Numbers and percentages of phlebotomines collected with black and white Shannon traps by species, sex and female/male ratio, and species richness (S). Xapuri municipality, Acre state, Brazil, August 2013-July 2015.

\begin{tabular}{|c|c|c|c|c|c|c|c|c|c|c|c|c|c|c|c|}
\hline \multirow{2}{*}{$\begin{array}{l}\text { Traps } \\
\text { Species } \\
\text { Sex and ratio } \\
\end{array}$} & \multicolumn{5}{|c|}{ White } & \multicolumn{5}{|c|}{ Black } & \multicolumn{5}{|c|}{ Total } \\
\hline & $F(d)$ & $M$ & Total & $F / M$ & $\%$ & $F(d)$ & $M$ & Total & $F / M$ & $\%$ & $F(d)$ & $M$ & Total & $F / M$ & $\%$ \\
\hline Brumptomyiina & & & & & & & & & & & & & & & \\
\hline $\begin{array}{l}\text { Br. pentacantha (Barretto) } \\
\text { Lutzomyiina }\end{array}$ & 4 & - & 4 & - & 0.10 & 3 & 1 & 4 & 3.00 & 0.20 & 7 & 1 & 8 & 7.00 & 0.12 \\
\hline Ev. bacula (Martins et al.) & - & - & - & - & - & - & 1 & 1 & - & 0.05 & - & 1 & 1 & - & 0.01 \\
\hline Ev. tarapacaensis (Le Pont et al.) & - & - & - & - & - & 1 & - & 1 & - & 0.05 & 1 & - & 1 & - & 0.01 \\
\hline Ev. saulensis (Floch \& Abonnenc) & $11(1)$ & - & 11 & - & 0.25 & 1 & - & 1 & - & 0.05 & 12 & - & 12 & - & 0.19 \\
\hline Ev. termitophila (Martins et al.), & $3(1)$ & - & 3 & - & 0.07 & - & - & - & - & - & 3 & - & 3 & - & 0.05 \\
\hline Lu. evangelistai Martins \& Fraiha & 1 & - & 1 & - & 0.02 & $2(1)$ & - & 2 & - & 0.10 & 3 & - & 3 & - & 0.05 \\
\hline Lu. sherlocki Martins et al. & $5(3)$ & - & 5 & - & 0.12 & $5(5)$ & - & 5 & - & 0.25 & 10 & - & 10 & - & 0.15 \\
\hline Lu. marinkellei* Young & 1 & 2 & 3 & 0.50 & 0.07 & 1 & - & 1 & - & 0.05 & 2 & 2 & 4 & 1.00 & 0.06 \\
\hline Mg. migonei (França) & 1(1) & - & 1 & - & 0.02 & - & 1 & 1 & - & 0.05 & 1 & 1 & 2 & 1.00 & 0.03 \\
\hline Pi. nevesi (Damasceno \& Arouck) & $17(2)$ & 2 & 19 & 8.50 & 0.44 & $7(4)$ & - & 7 & - & 0.35 & 24 & 2 & 26 & 12.00 & 0.41 \\
\hline Pi. serrana (Damasceno \& Arouck) & 1 & - & 1 & - & 0.02 & 2 & 2 & 4 & 1.00 & 0.20 & 3 & 2 & 5 & 1.50 & 0.08 \\
\hline Pr. choti (Floch \& Abonnenc) & 11 & 2 & 13 & 5.50 & 0.30 & 3 & 3 & 6 & 1.00 & 0.30 & 14 & 5 & 19 & 2.80 & 0.30 \\
\hline Sergentomyiina & & & & & & & & & & & & & & & \\
\hline Mi. trinidadensis (Newstead) & - & - & - & - & - & 2 & - & 2 & - & 0.10 & 2 & - & 2 & - & 0.03 \\
\hline Psychodopygina & & & & & & & & & & & & & & & \\
\hline Ny. antunesi (Coutinho) & $3(2)$ & 2 & 5 & 1.50 & 0.12 & $3(2)$ & 1 & 4 & 3.00 & 0.20 & 6 & 3 & 9 & 2.00 & 0.14 \\
\hline Ny. fraihai (Martins et al.) & - & - & - & - & - & $7(1)$ & - & 7 & - & 0.35 & 7 & - & 7 & - & 0.11 \\
\hline Ny. richardwardi (Ready \& Fraiha) & 1 & - & 1 & - & 0.02 & 1 & - & 1 & - & 0.05 & 2 & - & 2 & - & 0.03 \\
\hline Ny. shawi (Fraiha et al.) & $1,517(286)^{\mathrm{a}}$ & 111 & 1,628 & 13.66 & 37.81 & $585(165)$ & 53 & 638 & 11.03 & 31.85 & 2,102 & 164 & 2,266 & 12.81 & 36.00 \\
\hline Ny. whitmani (Antunes \& Coutinho) & $19(7)^{\mathrm{b}}$ & 3 & 22 & 6.33 & 0.51 & $4(3)$ & 4 & 8 & 1.00 & 0.40 & 23 & 7 & 30 & 3.28 & 0.45 \\
\hline Pa. aragaoi (Costa Lima) & 1 & - & 1 & - & 0.02 & - & - & - & - & - & 1 & - & 1 & - & 0.01 \\
\hline Pa. bigeniculata (Floch \& Abonnenc) & - & - & - & - & - & - & 1 & 1 & - & 0.05 & - & 1 & 1 & - & 0.01 \\
\hline Pa. dendrophyla (Mangabeira) & 1 & 1 & 2 & 1.00 & 0.04 & - & 1 & 1 & - & 0.05 & 1 & 2 & 3 & 0.50 & 0.05 \\
\hline Pa. pifanoi (Ortiz) & - & - & - & - & - & - & 1 & 1 & - & 0.05 & - & 1 & 1 & - & 0.01 \\
\hline Ps. amazonensis (Root) & 8 & 1 & 9 & 8.00 & 0.21 & 3(3) & - & 3 & - & 0.15 & 11 & 1 & 12 & 11.00 & 0.19 \\
\hline Ps. carrerai carrerai (Barretto) & $1,509(46)$ & 284 & 1,793 & 5.31 & 41.64 & $723(63)^{d}$ & 126 & 849 & 5.73 & 42.38 & 2,232 & 410 & 2,642 & 5.44 & 41.90 \\
\hline Ps. claustrei (Abonnenc et al.) & $3(1)$ & - & 3 & - & 0.07 & - & 1 & 1 & - & 0.05 & 3 & 1 & 4 & 3.00 & 0.06 \\
\hline Ps. davisi (Root) & $418(16)$ & 78 & 496 & 5.35 & 11.51 & $240(19)$ & 54 & 294 & 4.44 & 14.67 & 658 & 132 & 790 & 4.98 & 12.52 \\
\hline Ps. hirsutus hirsutus (Mangabeira) & $50(6)$ & 19 & 69 & 2.63 & 1.60 & $43(8)^{\mathrm{c}}$ & 8 & 51 & 5.37 & 2.55 & 93 & 27 & 120 & 3.44 & 1.90 \\
\hline Ps. lainsoni Fraiha \& Ward & $34(8)$ & 3 & 37 & 11.33 & 0.85 & $12(10)$ & 2 & 14 & 6.00 & 0.70 & 46 & 5 & 51 & 9.20 & 0.81 \\
\hline Ps. llanosmartinsi Fraiha \& Ward & $52(9)$ & 7 & 59 & 7.42 & 1.40 & $36(12)$ & 7 & 43 & 5.14 & 2.15 & 88 & 14 & 102 & 6.28 & 1.61 \\
\hline Ps. paraensis (Costa Lima) & 2 & - & 2 & - & 0.04 & - & - & - & - & - & 2 & - & 2 & - & 0.03 \\
\hline Ps. sp. (Guyanensis Series) & $2(1)$ & - & 2 & - & 0.04 & 2 & - & 2 & - & 0.10 & 4 & - & 4 & - & 0.06 \\
\hline Ps. sp. (Chagasi series) & 1 & - & 1 & - & 0.02 & - & - & - & - & - & 1 & - & 1 & - & 0.01 \\
\hline Th. auraensis (Mangabeira) & - & 29 & 29 & - & 0.70 & - & 15 & 15 & - & 0.75 & - & 44 & 44 & - & 0.70 \\
\hline Th. octavioi* (Vargas) & - & 28 & 28 & - & 0.65 & - & 5 & 5 & - & 0.25 & - & 33 & 33 & - & 0.52 \\
\hline
\end{tabular}


of $N y$. shawi (Data to be published). A nematode larva was detected in the head of one female of Ps. carrerai carrerai and is under investigation by specific molecular diagnosis.

\section{Discussion}

There are few studies on the attraction of phlebotomines by color. However, it is known that in this type of analysis, other factors must be taken into account, such as the environment chosen for the collections, the kind of light source, and the presence of human beings. This is because people also attract these insects, as demonstrated by Perez et al. [53] and Galati et al. [25]. In addition, the use of different traps in entomological surveys may reveal the diversity of species and their characteristic behavior.

The first study using black and white traps in Brazil was undertaken by Galati et al. [25] on the Bodoquena Plateau, midwestern Brazil, where Lu. almerioi Galati \& Nunes 1999, an anthropophilic species, was significantly more attracted to the black Shannon trap than to the white trap. Recently, studies on the nictemeral rhythm of phlebotomines in a craggy region near the Bodoquena Plateau also found a significant attractiveness of black Shannon traps to Lu. dispar Martins \& Silva, 1963, another anthropophilic species [38]. These two regions are speleological areas and both species are troglophyles, which may explain their significant attraction to the black Shannon traps.

The attractiveness of black and white Shannon traps to Ny. neivai (Pinto 1926) and Ny. intermedia (Lutz \& Neiva 1912) has also been investigated in agricultural and smallholding areas of the Ribeira Valley, São Paulo state, Brazil. The authors discovered that females of $\mathrm{Ny}$. intermedia were more attracted by the black Shannon trap and those of Ny. neivai by the white $[23,24]$. In another survey conducted in the Serra da Cantareira, metropolitan region of São Paulo, it was found that females of Pintomyia fischeri (Pinto 1926) are more attracted to the black Shannon trap, and those of Migonemyia migonei to the white. Based on these observations, it was suggested by the authors that $N y$. intermedia and Pi. fischeri may be better adapted to shady and humid locations within forested environments, while $\mathrm{Ny}$. neivai and $\mathrm{Mg}$. migonei might be more adapted to the anthropic environment [23, 48].

In the central area of Iran, Hesam-Mohammedi et al. [36] demonstrated the efficacy of different methods for sampling phlebotomine sandflies and verified the more numerous presence of Phlebotomus papatasi (Scopoli, 1786) and Phlebotomus sergenti Parrot, 1917 in black Shannon traps and Sergentomyia sintoni Pringle, 1953 in the white. Overall, more specimens were collected in the black trap.

In our study, Psychodopygus predominated over other groups in both black $(62.8 \%)$ and white $(57.4 \%)$ traps, with the females accounting for $84 \%$ of this genus. These findings are consistent with the results of Castellón et al. [13, 14], Cabanillas et al. [11], and Souza et al. [66], who also used white Shannon traps and human bait in Brazilian and Peruvian Amazonia, and found a high density of Psychodopygus females. These results differ from those of Alves et al. [2] who collected more males than females, with Psathyromyia 
Table 2. Comparison of the most frequent species of phlebotomines by sex and color of Shannon traps. Values of $p$ and $U$ by Mann-Whitney test (0.95 level of confidence). Xapuri municipality, Acre state, Brazil, August 2013-July 2015.

\begin{tabular}{|c|c|c|c|c|c|c|}
\hline \multirow{3}{*}{$\begin{array}{c}\text { Comparison } \\
\text { Traps } \\
U \text { and } p \text { values }\end{array}$} & \multicolumn{4}{|c|}{ By sex } & & \\
\hline & \multicolumn{2}{|c|}{ Black } & \multicolumn{2}{|c|}{ White } & \multicolumn{2}{|c|}{ By black and white colo } \\
\hline & $U$ & $p$ & $U$ & $p$ & $U$ & $p$ \\
\hline \multicolumn{7}{|l|}{ Species } \\
\hline Ny. shawi & 45.0 & 0.02 & 59.5 & 0.00 & 654 & 0.16 \\
\hline Ny. whitmani & 67.0 & 0.93 & 21 & 0.00 & 198 & 0.03 \\
\hline Pi. nevesi & 5.0 & 0.17 & 3 & 0.08 & 5 & 0.17 \\
\hline Pr. choti & 14.5 & 0.73 & 8 & 0.15 & 45 & 0.09 \\
\hline Ps. carrerai carrerai & 127.0 & 0.05 & 133 & 0.07 & 701.5 & 0.34 \\
\hline Ps. davisi & 100.5 & 0.11 & 72 & 0.01 & 366.5 & 0.01 \\
\hline Ps. hirsutus hirsutus & 57.5 & 0.04 & 52.5 & 0.03 & 321.5 & 0.22 \\
\hline Ps. lainsoni & 46.0 & 0.12 & 38 & 0.02 & 281 & 0.87 \\
\hline Ps. llanosmartinsi & 42.5 & 0.02 & 36.5 & 0.01 & 330.5 & 0.90 \\
\hline
\end{tabular}

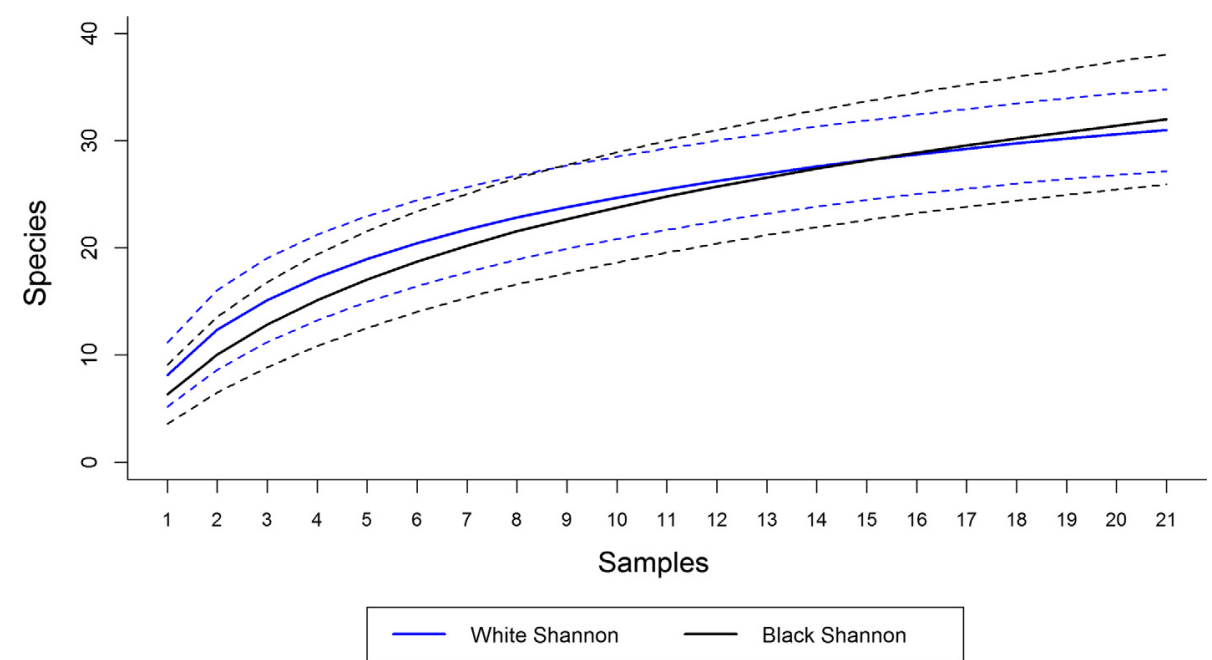

Figure 3. Sample-based species accumulation curves with a $95 \%$ confidence interval for white and black Shannon traps, August 2013-July 2015, Xapuri municipality, Acre state, Brazil.

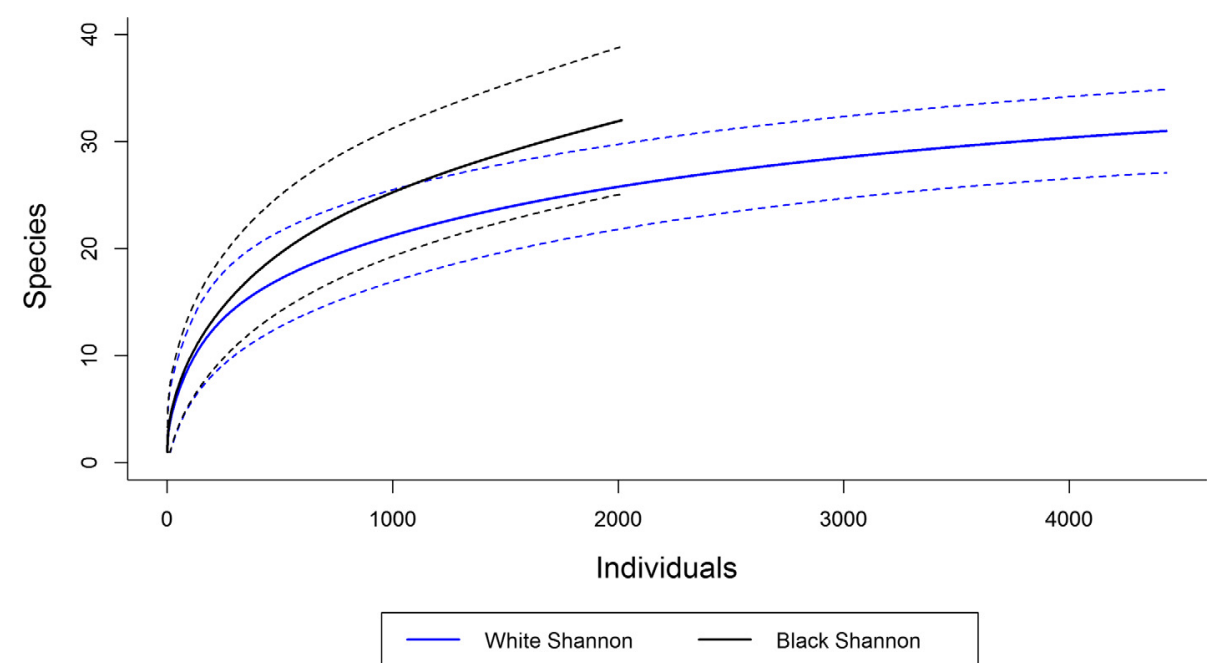

Figure 4. Individual-based species accumulation curves with a 95\% confidence interval for white and black Shannon traps, August 2013July 2015, Xapuri municipality, Acre state, Brazil. 

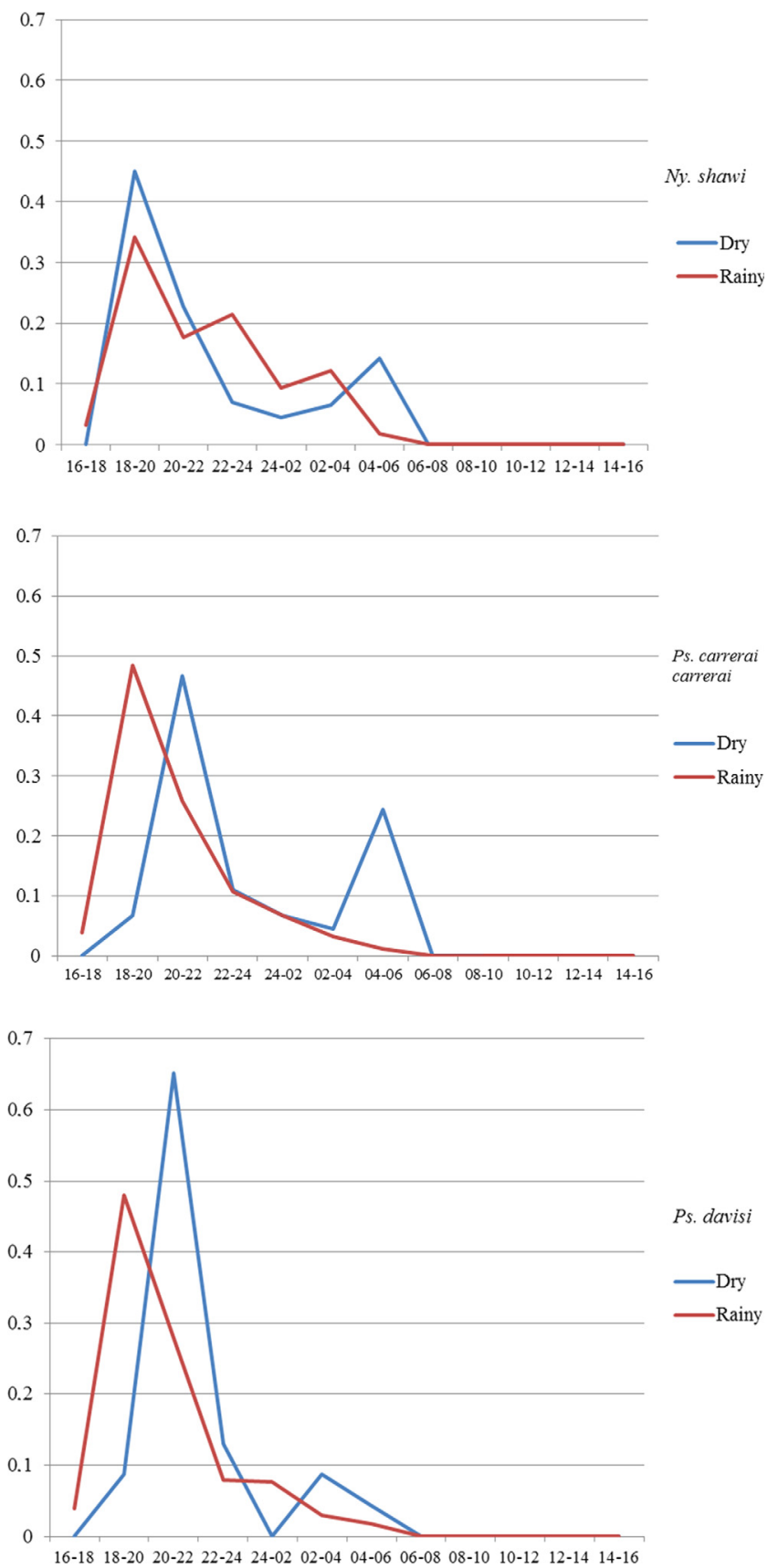

Figure 5. Hourly rhythm of the three most frequent species of phlebotomines by dry and rainy season, in March and August 2014, April and July 2015, in 24-h collections, Xapuri municipality, Acre state, Brazil.

dreisbachi, Ps. davisi, and Trichopygomyia trichopyga predominating. Another study with Shannon and CDC traps in the Amazonian biome, and in a transitional area between this system and the Andean biome in Colombia (Putumayo Department), collected $92.5 \%$ of the specimens in Shannon traps. Among the specimens collected, only $12 \%$ belonged to Psychodopygus, though the females also predominated at $86 \%$ [7]. In this same study, Nyssomyia accounted for $80 \%$ of the specimens, with the females representing $84 \%$. These findings differ from our results obtained in Xapuri, where Nyssomyia was the second most predominant group, accounting for $36.6 \%$, with the females contributing $92.5 \%$ of the specimens.

The phlebotomine fauna of the Acre state is rich and diverse, with 89 species so far recorded [27]. This number is being increased by two: $L u$. marinkellei and Th. octavioi in this report. There are few studies on phlebotomine fauna concerning richness analysis. Results show that high rates of richness and diversity are generally linked to more preserved environments with less anthropic action, as observed by Carvalho et al. [12] in Rio de Janeiro (RJ) and Saraiva et al. [59] in Minas Gerais (MG). Moreover, Feitosa et al. [20], comparing rural and urban environments in Santarém, Pará state, demonstrated by confidence intervals of individual-based rarefaction curves for each area, that urban sites presented the lowest expected number of species. Those of the rural sites attain higher expected values, with fewer individuals. Similar tendencies were observed for white and black traps in our study, which suggests that the black color is more attractive to species with a higher degree of dependence on preserved microenvironments than the white color.

For Xapuri, the curve of species accumulation did not stabilize, which indicates that new species will appear as the collections are made. More collections by Shannon traps are required, as well as with other trap modalities to verify the diversity and richness of species of the locality.

Comparing the numbers of specimens attracted to the traps between the two periods August to July 2013-2014 and August to July 2014-2015, it was observed that a greater number of specimens were collected in the former period. Although the Mann-Whitney test did not show any significant difference between them, based on the results of nine months with collections in both periods, it must be emphasized that the rainy season of 2014-2015 was much more intense than that of 2013-2014, with a historic flood in the state of Acre, including Xapuri where the Acre River borders the municipality. The flood reached a height of $17.62 \mathrm{~m}$ while the overflow level is $13.40 \mathrm{~m}$ [29], suggesting that this large volume of rainfall may have contributed to the decrease in the phlebotomine population.

The species of the Nyssomyia genus collected have already been reported in Acre, Brazil and neighboring countries [5, 10, $11,27,63,70,71]$. Nyssomyia whitmani is considered a species complex that is widespread in Brazil and is of epidemiological importance as the main vector of Leishmania ( $V$.) braziliensis in this country, and for having been incriminated in the transmission cycle of $L$. (V) guyanensis and $L$. (V.) shawi in the Amazon region [16, 22, 41, 42, 55]. In this study, this species was more attracted to the white Shannon trap, exactly as has been found in an area in the north of Paraná state [17]. This was different from the results obtained by Galati et al. [25] in areas close to caves in Mato Grosso do Sul state, where it was more frequent in the black trap.

Nyssomyia shawi has wide geographical distribution in the Brazilian Amazon, as well as in Bolivia, a country bordering the study region. In Bolivia, it is considered to be a vector of the agents of leishmaniasis due to its great abundance and has been found naturally infected by Leishmania (V.) braziliensis and L. (V.) guyanensis [10, 28, 31, 49]. 


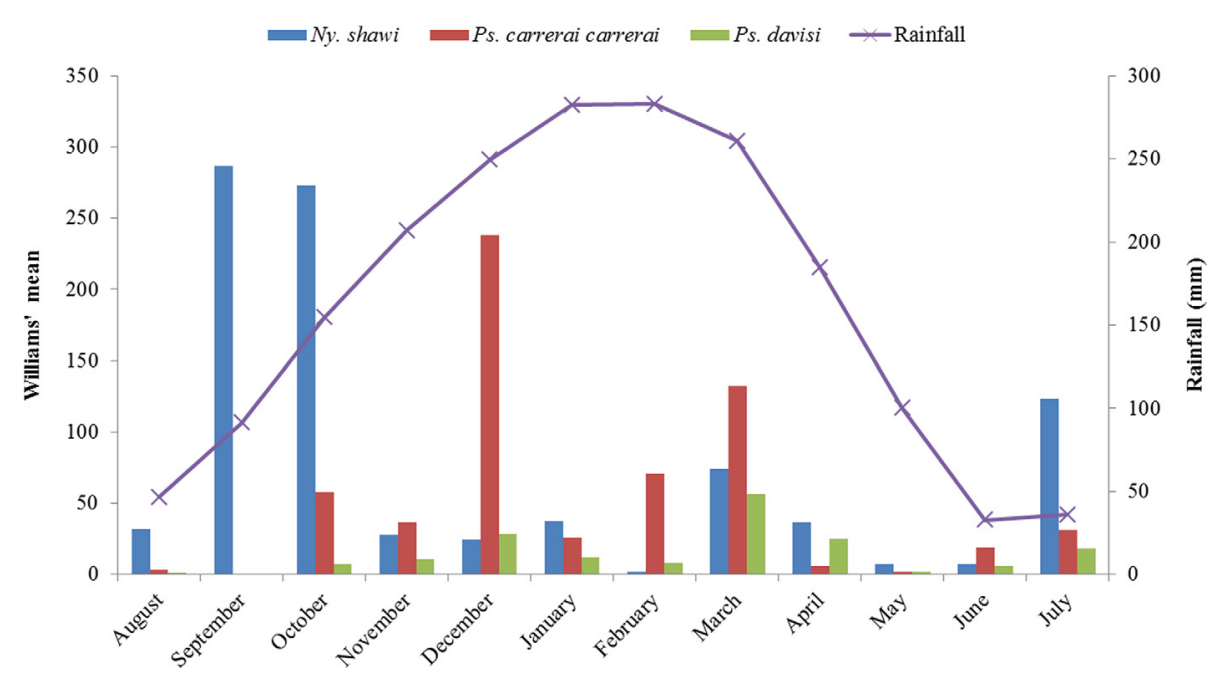

Figure 6. Monthly distribution of Williams' mean of the three most frequent species in Xapuri municipality and monthly mean rainfall (mm) in Rio Branco municipality, Acre state, Brazil, August 2013-July 2015.

The captures of this phlebotomine, with high frequencies in the present study, showed no statistically significant difference in the attractiveness to the two traps tested, and also presented a high density of this insect in collections with white traps [26]. Thus, bearing in mind that Shannon traps tend to capture anthropophilic species, the occurrence of high frequencies of Ny. shawi in our study, and the finding of specimens with natural infection by flagellates, reinforces these authors' points of view.

The Psychodopygus genus is rich (40 species described so far) and is widely distributed in the Amazon [27, 42]. In this research, Ps. carrerai carrerai and PS. davisi, both considered vectors of ACL agents in Brazil, were the dominant species. Highlighting the attractiveness of the white trap to Ps. davisi, this species' anthropophilic behavior and high density in white Shannon traps have been observed in other locations [30, 31, 66, 67]. Psychodopygus carrerai carrerai has been collected in both traps and is associated with primary forest environments. It has been reported naturally infected with Leishmania (V.) braziliensis, with massive infections in the digestive tract, in research carried out in Bolivia [43, 44]. More often, in northern Brazil, the species Ps. davisi has been found infected with L. (V.) braziliensis, L. (V.) guyanensis, L. (V.) naiff, and L. (V.) lainsoni [29, 61, 64, 65, 67], and is considered a potential vector in Rondônia [31] and Pará states [67]. These findings therefore provide strong evidence that these species may also be the vectors in the study area.

In this study, Ps. hirsutus hirsutus was found naturally infected by flagellates. Natural infections by trypanosomatids in this species have also been reported by Rangel et al. [56] in Minas Gerais and by Gil et al. [31] in Rondônia, and these infections were attributed by them to $L$. (V.) naiffi. Recently, this sandfly was found to be infected in Pará state. The isolated parasites were identified by monoclonal antibody testing as L. (V.) naiff by Souza et al. [66], who suggested that this phlebotomine may be a vector of $L$. (V.) naiffi in forested areas.

The other species of Psychodopygus were less abundant. However, they may have some epidemiological significance because they have been implicated as vectors of Leishmania in various locations in northern Brazil and border areas $[2,28,30,34,70]$.

Regarding the species of the genus Trichophoromyia, they are abundant in northern Brazil and neighboring countries. In Acre state and a neighboring country, Peru, Th. auraensis has been found in high densities in forest and peri/ intradomicilar environments [3, 5, 64], and has been reported as naturally infected by $L$. (V.) braziliensis, L. (V.) guyanensis, and Leishmania spp. [69, 70]. These observations may suggest that Th. auraensis has an important role in Leishmania transmission. Concerning Th. octavioi and the recently described Th. ruifreitasi [50], little is known about their behavior or about their females, herein identified as Trichophoromyia sp., because they are morphologically indistinguishable. However, in Xapuri municipality, in our previous studies using CDC traps, there has been a predominance of species of the genus Trichophoromyia (based on a sample of 11,295 specimens), while in this study the number of specimens attracted by Shannon traps was low.

The other species only collected in this study in the two traps in small numbers have been reported in other studies using CDC light traps in Acre state [3, 5, 69]. However, we highlight $\mathrm{Pa}$. pifanoi which has recently been resurrected from the synonymy of $P a$. shannoni by Sábio et al. [58] who considered $P a$. cuzquena to be the former's junior synonym. The female of this species has been described from specimens collected in the same locality as this study, but using CDC light traps.

According to Moschin et al. [48], the hourly rate may determine the time when the vector-host interaction occurs. In this study, the sandflies' activity peaks occurred between 18 and $20 \mathrm{~h}$ and 04 and $06 \mathrm{~h}$, the same times during which rubber and Brazil-nut extractors work and tourists hike in the area, exposing them to contact with the phlebotomine vectors. In other ACL endemic areas, the nocturnal activity of species of the genera Psychodopygus and Nyssomyia was observed, with peaks at 18-20 h, 22-00 h, and 04-06 h [34]. In Bolivian 
forests, the peak of $N y$. shawi activity has been recorded from 19 to $20 \mathrm{~h}$, a result similar to our findings. It is noteworthy that in this study no phlebotomine activity was observed during the day. However, in some northern regions of Brazil this habit has been registered with regard to some species of the genus Psychodopygus, such as Ps. wellcomei, Ps. complexus, and Ps. llanosmartinsi $[34,72]$.

Concerning the rainfall variable, it seems to influence the populations of Ps. carrerai carrerai significantly. In the rainy months, Ps. carrerai carrerai and Ps. davisi tend to appear in the first few hours of collection, as from $18 \mathrm{~h}$. While Ny. shawi appears equally in both periods, but with predominance in the dry months. In other Brazilian Amazonian studies, rainfall and humidity contribute significantly to populations of phlebotomines of the genus Psychodopygus [34, 72]. In this study, although rainfall had no significant correlation with the frequency of $N y$. shawi, there was a high population density of this species in the dry months. This result is similar to observations for other species of the genus Nyssomyia in other Brazilian localities [9, 34].

In the Amazon region, the rate of infection by flagellates in phlebotomine flies is high, the most common genera being Psychodopygus and Nyssomyia, with a great variety of vector species of Leishmania. This same scenario was repeated in this study, including the finding of females naturally infected by flagellates of Leishmania sp., corroborating the results obtained in other areas of the Brazilian Amazon basin [4, 63, 66].

Here we report the finding of a female specimen of Ps. carrerai carrerai with the presence of a nematode, some of which live a part of their life cycle in sandflies [6]. In the Old World, some infestations have been reported in sandflies, some examples of which are Madathamugadia wanjii in Phlebotomus duboscqi [6], Didilia sp., Didilia ooglypta, Psychodiella mackiei and Tylenchoidea super family in Phlebotomus papatasi and Phlebotomus sergenti [18, 40, 47, 62, 68], Steinernematidae family in Phlebotomus tobbi [39]. In the Americas, experimental infestations by Anandarema phlebotophaga and other nematodes have been observed in Colombia and Brazil [54, 60]. In Argentina, wild populations of Pi. fischeri have been found parasitized by Tylenchid nematodes [21].

Keeping in mind that Shannon traps tend to attract more anthropophilic species than other types of traps, such as CDC light traps, and that some species were attracted only to the black trap and others only to the white trap, we suggest the combined use of traps of the two colors for a more complete evaluation of the richness, diversity, and anthropophily of phlebotomine fauna.

\section{Conflict of interest}

The authors have no potential conflict of interest.

Acknowledgements. The authors wish to express their thanks to: Mrs. Carmelinda Gonçalves and Thayna de Souza of the Health Secretariat of the State of Acre - SESACRE; to Mr. Joaquim Vidal of the municipal Surveillance Secretariat of Xapuri for his logistic support. We also thank Fundação de Amparo à Pesquisa do Estado do Acre (FAPAC) (Grant Agreement Number PPSUS No. 774445) for the financial support and the National Council for Scientific and Technological Development (CNPq) for the scholarship granted to Brilhante AF.

\section{References}

1. Acre. Governo do Estado do Acre. [cited 2016 March 21]. Available from: http://www.ac.gov.br/wps/portal/acre/Acre/ estado-acre/municipios.

2. Alves VR, Freitas RA, Santos FL, Oliveira AFJ, Barrett TV, Shimabukuro PHF. 2010. Sand flies (Diptera, Psychodidae, Phlebotominae) from Central Amazonia and four new records for the Amazonas state, Brazil. Revista Brasileira de Entomologia, 56, 220-227.

3. Araujo-Pereira T, Fuzari AA, Andrade Filho JD, Pita-Pereira D, Britto C, Brazil RP. 2014. Sand fly fauna (Diptera: Psychodidae: Phlebotominae) in an area of leishmaniasis transmission in the municipality of Rio Branco, state of Acre, Brazil. Parasites \& Vectors, 7, 2-5.

4. Arias JR, Freitas RA. 1982. The known geographical distribution of sand flies in the state of Acre, Brazil (Diptera: Psychodidae). Acta Amazônica, 12, 401-408.

5. Azevedo ACR, Costa SM, Pinto MCG, Souza JL, Cruz HC, Vidal J, Rangel EF. 2008. Studies on the sandfly fauna (Diptera: Psychodidae: Phlebotominae) from transmission areas of American cutaneous leishmaniasis in state of Acre, Brazil. Memórias do Instituto Oswaldo Cruz, 103, 760-767.

6. Bain O, Petit G, Paperna I, Finkelman S, Killick-Kendrick M. 1992. A new filarial of lizard transmitted by sandflies. Memórias do Instituto Oswaldo Cruz, 87, 21-29.

7. Barreto M, Burbano ME, Barreto P. 2000. Lutzomyia sand flies (Diptera: Psychodidae) from middle and lower Putumayo Department, Colombia, with new records to the country. Memórias do Instituto Oswaldo Cruz, 95, 633-639.

8. Browne SM, Bennett GF. 1981. Response of mosquitoes (Diptera: Culicidae) to visual stimuli. Journal of Medical Entomology, 18, 505-521.

9. Brilhante AF, Dorval MEC, Galati EAB, Cristaldo G, Rocha HC, Nunes VLB. 2015. Phlebotomine fauna (Diptera: Psychodidae) in an area of fishing tourism in CentralWestern Brazil. Revista do Instituto de Medicina Tropical, 57, 233-238.

10. Bustamante M, Díaz M, Espinoza J, Parrado R, Reithinger R, García AL. 2012. Sand fly fauna in Chapare, Bolivia: an endemic focus of Leishmania (Viannia) braziliensis. Journal of Medical Entomology, 49, 1159-1162.

11. Cabanillas M, Braga J, Viena M. 2001. Flebotomíneos da floresta de Terra Firme da Amazonia Peruana (Diptera: Psychodidae). Acta Amazônica, 31, 275-284.

12. Carvalho BM, Maximo M, Costa WA, De Santanta ALF, Da Costa SM, Da Costa Rego TAN, Pita-Pereira D, Rangel EF. 2013. Leishmaniasis transmission in an ecotourism area: potential vectors in Ilha Grande, Rio de Janeiro state, Brazil. Parasites \& Vectors, 6, 325.

13. Castellón EG, Arias JR, Freitas RA, Naiff RD. 1994. Os flebotomíneos da região Amazônica, estrada Manaus Humaitá, estado do Amazonas, Brasil (Diptera: Psychodidae: Phlebotominae). Acta Amazônica, 24, 91-102.

14. Castellón EG, Fé NF, Buhrnheim PF, Fé FA. 2000. Flebotomíneos (Diptera: Psychodidae) na Amazônia. II. Listagem das espécies coletadas na Bacia Petrolífera no Rio Urucu, 
Amazonas, Brasil, utilizando diferentes armadilhas e iscas. Revista Brasileira de Zoologia, 17, 455-462.

15. Colwell RK, Mao CX, Chang J. 2004. Interpolating, extrapolating, and comparing incidence-based species accumulation curves. Ecology, 85, 2717-2727.

16. Costa SM, Cechinel M, Bandeira V, Zannuncio JC, Lainson R, Rangel EF. 2007. Lutzomyia (Nyssomyia) whitmani s.l. (Antunes \& Coutinho 1939) (Diptera: Psychodidae: Phlebotominae): geographical distribution and the epidemiology of American Cutaneous Leishmaniasis in Brazil. Minireview. Memórias do Instituto Oswaldo Cruz, 102, 149-153.

17. Cruz CFR, Cruz MFR, Galati EAB. 2013. Sandflies (Diptera: Psychodidae) in rural and urban environments in an endemic area of cutaneous leishmaniasis in southern Brazil. Memórias do Instituto Oswaldo Cruz, 108, 303-311.

18. Dinesh DS, Kumar V, Das P. 2013. Infestation of Nematodes in Phlebotomus argentipes Annandale and Brunetti (Diptera: Psycodidae [sic]), Bihar, India. Global Journal of Medical Research, 13, 7-9.

19. Duarte AF. 2006. Aspectos da climatologia do Acre, Brasil, com base no intervalo de 1971-2000. Revista Brasileira de Meteorologia, 3, 308-317.

20. Feitosa MAC, Julião GR, Costa MDP, Belém B, Pessoa FAC. 2012. Diversity of sand flies in domiciliary environment of Santarém, state of Pará, Brazil: species composition and abundance patterns in rural and urban areas. Acta Amazônica, $42,507-514$.

21. Fernández MS, Santini MS, Diaz JI, Villarquide L, Lestani E, Solomón OD, Achinelly M. 2016. Parasitism by tylenchid nematodes in natural populations of Pintomyia fischeri (Diptera: Psychodidae: Phlebotominae) in Argentina. SM Tropical Medicine Journal, 1, 1001.

22. Freitas RA, Naiff RD, Barrett TV. 2002. Species diversity and flagellate infections in the sand fly fauna near Porto Grande, state of Amapá, Brazil (Diptera: Psychodidae. Kinetoplastida: Trypanosomatidae). Memórias do Instituto Oswaldo Cruz, 97, 53-59.

23. Galati EAB, Marassá AM, Fonseca MB, Gonçalves-Andrade RM, Consales CA, Bueno EFM. 2010. Phlebotomines (Diptera, Psychodidae) in the Speleological Province of the Ribeira Valley: 3. Serra district - area of hostels for tourists who visit the Parque Estadual do Alto Ribeira (PETAR), state of São Paulo, Brazil. Revista Brasileira de Entomologia, 54, 665-676.

24. Galati EAB, Marassá AM, Gonçalves-Andrade RM, Bueno EFM, Paiva BM, Malafronte RS. 2010. Nyssomyia intermedia (Lutz \& Neiva) and Nyssomyia neivai (Pinto) (Diptera, Psychodidae, Phlebotominae) in a sympatric area: seasonal and nocturnal hourly rhythm in black and white modified Shannon traps. Revista Brasileira de Entomologia, 54, 677-686.

25. Galati EAB, Nunes VLB, Dorval MEC, Cristaldo G, Rocha HC, Gonçalves-Andrade RM, Naufel G. 2001. Attractiveness of black Shannon trap for phlebotomines. Memórias do Instituto Oswaldo Cruz, 96, 641-647.

26. Galati EAB. 2003. Classificação de Phlebotominae, in Flebotomíneos do Brasil. Rangel EF, Lainson R, Editors. Ed. Fiocruz: Rio de Janeiro. p. 23-51.

27. Galati EAB. 2016. Phlebotominae (Diptera, Psychodidae): classificação, morfologia, terminologia e identificação de adultos [apostila], vol. 1, USP: São Paulo.

28. García AL, Tellez LT, Parrado R, Rojas E, Bermudez H, Dujardin JC. 2007. Epidemiological monitoring of American tegumentary leishmaniasis: molecular characterization of a peridomestic transmission cycle in the Amazonian lowlands of Bolivia. Transactions of Royal Society Tropical Medicine and Hygiene, 101, 1208-1213.

29. Gazeta do Acre. Especial: Uma das piores enchentes da história do Acre. Available from: http://agazetadoacre.com/especialuma-das-piores-enchentes-da-historia-do-acre/ (dated 02-032015; retrieved 11-05-2017).

30. Gil LHS, Araújo MS, Villalobos JM, Camargo LMA, Ozaki LS, Fontes CJF, Ribolla PEM, Katsuragawa TH, Cruz RM, Almeida e Silva A, Silva LHP. 2009. Species structure of sand fly (Diptera: Psychodidae) fauna in the Brazilian western Amazon. Memórias do Instituto Oswaldo Cruz, 104, 955-959.

31. Gil LHS, Basano SA, Souza AA, Silva MGS, Barata I, Ishikawa EA, Camargo LMA, Shaw JJ. 2003. Recent observations on the sand fly (Diptera: Psychodidae) fauna of the state of Rondônia, Western Amazônia, Brazil: the importance of Psychodopygus davisi as a vector of zoonotic cutaneous leishmaniasis. Memórias do Instituto Oswaldo, 98, 751-755.

32. Gilbert IH, Gouck HK. 1957. Influence of surface color on mosquito landing rates. Journal of Economic Entomology, 50, 678-680.

33. Godoy RE, Galati EAB. 2016. Revalidation of Nyssomyia fraihai (Martins, Falcão \& Silva 1979) (Diptera: Psychodidae). Journal of Medical Entomology, 53, 1303-1311.

34. Godoy RE, Santana ALF, Graser C, Rangel EF, Vilela ML. 2016. Aspects on the ecology of phlebotomine sand flies (Diptera: Psychodidae) from Guaraí, state of Tocantins, Brazil, endemic area for American cutaneous leishmaniasis. Journal of Medical Entomology, 54, 229-235.

35. Haddow AJ. 1960. Studies on the biting habits and medical importance of East African mosquitos in the genus Aedes. I Subgenera Aedimorphus, Banksinella and Dunnius. Bulletin of Entomology Research, 50, 759-779.

36. Hesam-Mohammadi M, Rassi Y, Abai MR, Akhavan AA, Rafizadeh S, Sanei Dehkordi A, Sharafkhah M. 2014. Efficacy of different sampling methods of sand flies (Diptera: Psychodidae) in endemic focus of cutaneous leishmaniasis in Kashan district, Isfahan province, Iran. Journal of Arthropod-Borne Disease, 8, 156-162.

37. IBGE - Instituto Brasileiro de Geografia e Estatística. 2016. Anuário Estatístico do Brasil [Internet], [cited 2016 October 16]. Available from: www.ibge.gov.br.

38. Infran JOM, Souza DA, Fernandes WS, Casaril AE, Eguchi GU, Oshiro ET, Fernandes FES, Paranhos Filho AC, Oliveira AG. 2017. Nycthemeral rhythm of phlebotominae (Diptera: Psychodidae) in a craggy region, transitioning between the Wetland and the Plateau, Brazil. Journal of Medical Entomology, 54, 114-124.

39. Karakus M, Arserim SK, Töz SÖ, Özbel Y. 2013. Detection of entomopathogen nematode [EPN-Sand Flies (Phlebotomus tobbi)] caught in the wild in Aydin, Kusadasi town and its assessment as a biological control agent. Türkiye Parazitoloji Dergisi, 37, 36-39.

40. Killick-Kendrick R, Killick-Kendrick M, Qala I, Nawi NA, Ashaford RW, Tang Y. 1989. Preliminary observations on a tetradonematid nematode of phlebotomine sandflies of Afghanistan. Annales de Parasitologie Humaine et Comparée, 64, 332-339.

41. Lainson R, Shaw JJ, Silveira FT, de Souza AAA, Braga RR, Ishikawa EAY. 1994. The dermal leishmaniases of Brazil, with 
special reference to the eco-epidemiology of the disease in Amazonia. Memórias do Instituto Oswaldo Cruz, 89, 435-443.

42. Lainson R, Shaw JJ. 2005 New World leishmaniasis, in Microbiology and Microbial Infections, Parasitology. Topley \& Wilson's. Cox FEG, Kreir JP, Wakelin D, Editor. Arnold: Sydney, Auckland. p. 313-349.

43. Le Pont F, Breniere RS, Mouchet J, Desjeux P. 1988. Leishmaniose en Bolivie. III. Psychodopygus carrerai carrerai (Barretto, 1946) nouveau vecteur de Leishmania braziliensis braziliensis en milieu sylvatique de région subandine basse. Comptes Rendus de l'Académie des Sciences - Séries III, 307, 279-282.

44. Le Pont F, Mouchet J, Desjeux P. 1989. Leishmaniasis in Bolivia - VI. Observations on Lutzomyia nuneztovari anglesi Le Pont \& Desjeux, 1984 the presumed vector of tegumentary leishmaniasis in the Yungas focus. Memórias do Instituto Oswaldo Cruz, 84, 277-278.

45. Marcondes CB. 2007. A proposal of generic and subgeneric abbreviations of phlebotomines sandflies (Diptera: Psychodidae: Phlebotominae) of the world. Entomology News, 118, 351-356.

46. Maroli M, Feliciangeli MD, Arias J. 1997. Metodos de Captura, Conservacion y Montaje de los Flebotomos (Diptera: Psychodidae). Documento OPS/HCP/ HCT/95/97, Organizacion Panamericana de la Salud, Washington DC, USA. p. 72.

47. Missiroli A. 1932. Sullo sviluppo di una gregarina del Phlebotomus. Annali di Igiene, 42, 373-377.

48. Moschin JC, Ovallos FG, Sel IA, Galati EAB. 2013. Ecological aspects of phlebotomine fauna (Diptera, Psychodidae) of Serra da Cantareira, Greater São Paulo Metropolitan region, state of São Paulo, Brazil. Revista Brasileira de Epidemiologia, 16, 190-201.

49. Ogawa GM, Pereira Júnior AM, Resadore F, Ferreira RGM, Medeiros JF, Camargo LMA. 2016. Sandfly fauna (Diptera: Psychodidae) from caves in the state of Rondônia, Brazil. Revista Brasileira de Parasitologia Veterinária, 25(1), 61-68.

50. Oliveira AFJ, Teles CBG, Medeiros FM, Camargo LMA, Pessoa FAC. 2015. Description of Trichophoromyia ruifreitasi, a new phlebotomine species (Diptera, Psychodidae) from Acre state, Brazilian Amazon. ZooKeys, 526, 65-73.

51. Paiva BR, Secundino NFC, Pimenta PFP, Galati EAB, Andrade-Júnior HF, Malafronte RS. 2007. Standardization of conditions for PCR detection of Leishmania spp. DNA in sandflies (Diptera, Psychodidae). Cadernos de Saúde Pública, 23, 87-94.

52. Pereira Júnior AM, Teles CBG, dos Santos APA, Rodrigues MD, Marialva EF, Pessoa FAC, Medeiros JF. 2015. Ecological aspects and molecular detection of Leishmania DNA Ross (Kinetoplastida: Trypanosomatidae) in phlebotomine sandflies (Diptera: Psychodidae) in terra firme and várzea environments in the Middle Solimões Region, Amazonas state, Brazil. Parasites \& Vectors, 8, 180.

53. Perez JE, Villaseca A, Llanos-Cuenta A, Campos M, Guerra H. 1988. Técnicas para colectar "titiras" (Lutzomyia spp., Diptera: Psychodidae), em ambientes alto andinos peruanos. Revista Peruana de Entomologia, 30, 77-80.

54. Poinar GO, Ferro C, Morales A, Tesh RB. 1993. Anandranema phlebotophaga $\mathrm{n}$. gen., n. sp. (Allantonematidae: Tylenchida), a new nematode parasite of phlebotomine sand flies (Psychodidae: Diptera) with notes on experimental infections of these insects with parasitic rhabditoids. Fundamental and Applied Nematology, 16, 11-16.
55. Queiroz RG, Vasconcelos IA, Vasconcelos AW, Pessoa FA, Souza RN, David JR. 1994. Cutaneous leishmaniasis in Ceará state in Northeasten Brazil: incrimination of Lutzomyia whitmani (Diptera: Psychodidae) as a vector of Leishmania braziliensis in Baturite municipality. The American Journal of Tropical Medicine and Hygiene, 50, 693-698.

56. Rangel EF, Ryan L, Lainson R, Shaw JJ. 1985. Observations on the sand fly (Diptera: Psychodidae) fauna of Além Paraiba, State of Minas Gerais, Brazil, and the isolation of a parasite of the Leishmania braziliensis complex from Psychodopygus hirsuta hirsuta. Memórias do Instituto Oswaldo Cruz, 80, 373-374.

57. Sabio PB, Andrade AJ, Galati EAB. 2014. Assessment of the taxonomic status of some species included in the Shannoni complex, with the description of a new species of Psathyromyia (Diptera: Psychodidae: Phlebotominae). Journal of Medical Entomology, 51, 331-341.

58. Sábio PB, Brilhante AF, Quintana MG, Andrade AJ, Galati EAB. 2016. On the synonyms of Psathyromyia (Psathyromyia) shannoni (Dyar, 1929) and Pa. bigeniculata (Floch \& Abonnenc, 1941) and the resuscitation of Pa. pifanoi (Ortiz, 1972) with the description of its female (Diptera: Psychodidae: Phlebotominae). Journal of Medical Entomology, 53, 1140-1147.

59. Saraiva L, Silva Reis A, Marteleto NRJ, Sampaio PAA, Rêgo FD, Lima ACVMR, Gontijo CM, Andrade-Filho JD. 2015. Survey of Sand Flies (Diptera: Psychodidae) in an environmentally protected area in Brazil. PLoS One, 10, $\mathrm{e} 0134845$.

60. Secundino NFC, Araújo MSS, Oliveira GHB, Massara CL, Carvalho OS, Lanfredi RM, Pimenta PFP. 2002. Preliminary description of a new entomoparasitic nematode infecting Lutzomyia longipalpis sand fly, the vector of visceral leishmaniasis in the New World. Journal of Invertebrate Pathology, 80, 35-40.

61. Shannon R. 1939. Methods for collecting and feeding mosquitos in jungle yellow fever studies. The American Journal of Tropical Medicine and Hygiene, 19, 131-148.

62. Shortt HE, Swaminath CS. 1927. Monocystis mackiei n. sp. parasitic in Phlebotomus argentipes, Ann. and Brun. The Indian Journal of Medical Research, 15, 539-553.

63. Silva TRR, Assis MDG, Freire MP, Rego FD, Gontijo CMF, Shimabukuro PHF. 2014. Molecular Detection of Leishmania in sand flies (Diptera: Psychodidae: Phlebotominae) collected in the Caititu indigenous reserve of the municipality of Labrea, state of Amazonas, Brazil. Journal of Medical Entomology, 51, $1-7$.

64. Silva-Nunes M, Cavasini CE, Silva NS, Galati EAB. 2008. Epidemiologia da leishmaniose tegumentar e descrição das populações de flebotomíneos no município de Acrelândia, Acre, Brasil. Revista Brasileira de Epidemiologia, 11, 241-251.

65. Silveira FT, Souza AAA, Lainson R, Shaw JJ, Braga RR, Ishikawa EEA. 1991. Cutaneous leishmaniasis in the Amazon Region: natural infection of the sandfly Lutzomyia ubiquitalis (Psychodidae: Phlebotominae) by Leishmania (Viannia) lainsoni in Pará state, Brazil. Memórias do Instituto Oswaldo Cruz, 86, 127-130.

66. Souza AAA, Santos TV, Jennings YLL, Ishikawa EAY, Barata IR, Silva MGS, Lima JAN, Shaw J, Lainson R, Silveira FT. 2016. Natural Leishmania (Viannia) spp. infections in phlebotomine sand flies (Diptera: Psychodidae) from the Brazilian Amazon region reveal new putative transmission cycles of American cutaneous leishmaniasis. Parasite, 23, 22. 
67. Souza AAA, Silveira FT, Lainson R, Barata IR, Silva MGS, Lima JAN, Pinheiro MSB, Silva FMM, Vasconcelos LS, Campos MB, Ishikawa EAY. 2010. Phlebotominae fauna in Serra dos Carajás, Pará state, Brazil, and its possible implications for the transmission of American tegumentar leishmaniasis. Revista Pan-Amazônica de Saúde, 1, 45-51.

68. Tang Y, Killick-Kendrick R, Hominick WM. 1997. Life cycle of Didilia ooglypta (Nematoda: Tetradonematidae), a parasite of phlebotomine sandflies of Afghanistan. Nematologia, 42, 491-503.

69. Teles CBG, Santos APA, Freitas RA, Oliveira AFJ, Ogawa GM, Rodrigues MS, Pessoa FAC, Medeiros JF, Camargo LMA. 2016. Phlebotomine sandfly (Diptera: Psychodidae) diversity and their Leishmania DNA in a hot spot of American cutaneous leishmaniasis human cases along the Brazilian border with Peru and Bolivia. Memórias do Instituto Oswaldo Cruz, 111, 423-432.

70. Valdívia HO, de los Santos MB, Fernández R, Baldeviano GC, Zorrilla VO, Vera H, Lucas CM, Edgel KA, Lescano AG, Mundal KD, Graf PCF. 2012. Natural Leishmania infection of
Lutzomyia (Trichophoromyia) auraensis in Madre de Dios, Peru, detected by a fluorescence resonance energy transferbased real-time polymerase chain reaction. The American Journal of Tropical Medicine and Hygiene, 87, 511-517.

71. Vilela ML, Pita-Pereira D, Azevedo CG, Godoy RE, Britto C, Rangel EF. 2013. The phlebotomine fauna (Diptera: Psychodidae) of Guaraí, state of Tocantins, with an emphasis on the putative vectors of American cutaneous leishmaniasis in rural settlement and periurban areas. Memórias do Instituto Oswaldo Cruz, 108, 578-585.

72. Ward RD, Shaw JJ, Lainson R, Fraiha H. 1973. Leishmaniasis in Brazil: VIII. Observations on the phlebotomine fauna of an area highly endemic for cutaneous leishmaniasis, in the Serra dos Carajás, Pará State. Transactions of Royal Society and Tropical Medicine and Hygiene, 67, 174-183.

73. Xapuri Info. Xapuri Socioambiental. Seringal Cachoeira: Ecoturismo entre as seringueiras. Available from: http:// www.xapuri.info/guiaxapuri/seringal-cachoeira-ecoturismoentreseringueiras/ (dated 05-10-2015).

Cite this article as: Brilhante AF, de Ávila MM, de Souza JF, Medeiros-Sousa AR, Sábio PB, de Paula MB, Godoy RE, Melchior LAK, Nunes VLB, de Oliveira Cardoso C \& Galati EAB: Attractiveness of black and white modified Shannon traps to phlebotomine sandflies (Diptera, Psychodidae) in the Brazilian Amazon Basin, an area of intense transmission of American cutaneous leishmaniasis. Parasite, $2017,24,20$.

\section{O PARASTE}

An international open-access, peer-reviewed, online journal publishing high quality papers on all aspects of human and animal parasitology

Reviews, articles and short notes may be submitted. Fields include, but are not limited to: general, medical and veterinary parasitology; morphology, including ultrastructure; parasite systematics, including entomology, acarology, helminthology and protistology, and molecular analyses; molecular biology and biochemistry; immunology of parasitic diseases; host-parasite relationships; ecology and life history of parasites; epidemiology; therapeutics; new diagnostic tools.

All papers in Parasite are published in English. Manuscripts should have a broad interest and must not have been published or submitted elsewhere. No limit is imposed on the length of manuscripts.

Parasite (open-access) continues Parasite (print and online editions, 1994-2012) and Annales de Parasitologie Humaine et Comparée (1923-1993) and is the official journal of the Société Française de Parasitologie. 